\title{
Preparation and Characterization of CA/GO Mixed Matrix Forward Osmosis Membranes
}

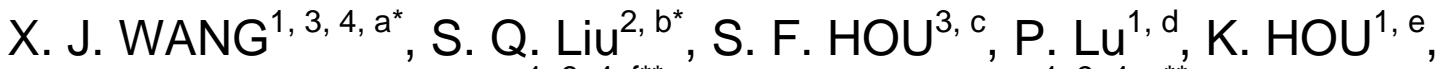 \\ Z. P. WANG ${ }^{1,3,4, f^{\star *}}$ and L. G. WANG ${ }^{1,3,4,9^{* *}}$ \\ ${ }^{1}$ School of Resources and Environment, University of Jinan, Jinan, China, 250022 \\ ${ }^{2}$ School of Chemistry and Chemical Engineering, University of Jinan, Jinan, China, 250022 \\ ${ }^{3}$ Shandong Provincial Engineering Technology Research Center for Ecological \\ Carbon Sink and Capture Utilization, Jinan, China, 250022 \\ ${ }^{4}$ Shandong Provincial Engineering Technology Research Center for Groundwater \\ Numerical Simulation and Contamination Control, Jinan, China, 250022 \\ achm_wangxj@ujn.edu.cn, bchm_liusq@ujn.edu.cn, 61965270@qq.com, d280667550@qq.com, \\ e1320418626@qq.com, ${ }^{\dagger}$ chm_wangzp@ujn.edu.cn, Igwang666@163.com \\ ${ }^{*}$ Co-authors, ${ }^{*}$ Corresponding Author
}

Keyword: Graphene oxide, Mixed matrix, Forward osmosis, Membrane

\begin{abstract}
In this paper, a novel mixed matrix forward osmosis (FO) membrane was fabricated by introducing graphene oxide ( $\mathrm{GO}$ ) into the cellulose acetate (CA)-based casting solution using phase inversion methods. The factors---GO content and blending temperature were tested for their effects on pure water flux and reverse salt flux of FO membranes. The results showed that the mixed matrix FO membrane prepared under optimized composition had excellent desalination performance (high pure water flux $=16.0 \mathrm{~L} / \mathrm{m}^{2} \cdot \mathrm{h}$ and the reverse salt flux was below $7 \mathrm{~g} / \mathrm{m}^{2} \cdot \mathrm{h}$ ). Moreover, SEM images showed that addition of GO resulted in mixed matrix FO membrane with a smoother surface. The contact angle of the membranes decreased from $71.6^{\circ}$ to $58.2^{\circ}$ with increase GO concentration from $0 \%$ to $0.15 \%$.
\end{abstract}

\section{Introduction}

FO is an osmotic pressure-driven membrane process in which water moves spontaneously across a semi-permeable membrane from the feed solution side to the draw solution side [1]. The early study of FO membranes employed reverse osmosis membranes of hydrophilic cellulose acetate (CA) and/or cellulose triacetate (CTA) polymers, which show low permeate water flux since their porous support layer [2]. However, higher internal concentration polarization was observed as well as, which occurs within the support layer and will cause a dramatic loss in the effective osmotic driving [3].

Recently, researchers have attracted considerable attention of synthesizing the membranes with nanoparticles to increase the membrane performance. Different nanomaterials such as graphene oxide, titanium dioxide, molecular sieve and carbon nanotube had been added to membrane structure for improving the properties of membrane such as pure water flux and anti-fouling [4-7]. Among the nanoparticles studied, graphene oxide (GO) is one of the most widely used material for preparing high-performance FO membranes due to their special properties such as unique structural properties, high chemical stability, strongly drophilicity and excellent antifouling properties[8-13]. This study aimed to prepare hydrophily mixed matrix FO membranes by incorporating the hydrophilic GO into the CA-based membrane.

\section{Experimental}

Materials. CA (ACROS ORGANICS) was used as the base polymer. Dimethylformamide (DMF, Tianjin Fuyu Chemical Co., Ltd.), acetone (Yantai Sanhe Chemical Co., Ltd.) were used as solvent, Polyethylene glycol (PEG400, Tianjin Kemiou Chemical Reagent Co., Ltd.) and GO (made in our Lab.) were used as the pore-foaming agent. And sodium chloride (Tianjin Bodi 
Chemical Co., Ltd.) and glucose (Xiwang Biochemical Technology Co., Ltd.) were used for membrane performance testing.

Membrane Preparation. For preparing the casting solution, different concentrations of GO were dispersed in DMF and then the solution was vibrated via ultrasonication for 1 hour until it became homogeneous. Afterward, a desired amount of acetone, PEG400 and CA were added. The solution was then stirred for 6 hours and keeping still overnight for degassing. The prepared dope was then cast on polyester mesh screen. After partial evaporation, the glass plate was immersed smoothly in a coagulation bath with suitable temperature.

Membrane Performance Testing. The prepared membrane performance was analyzed by a lab-scale device (Fig. 1) using a test cell with $27 \mathrm{~cm}^{2}$ effective membrane area.

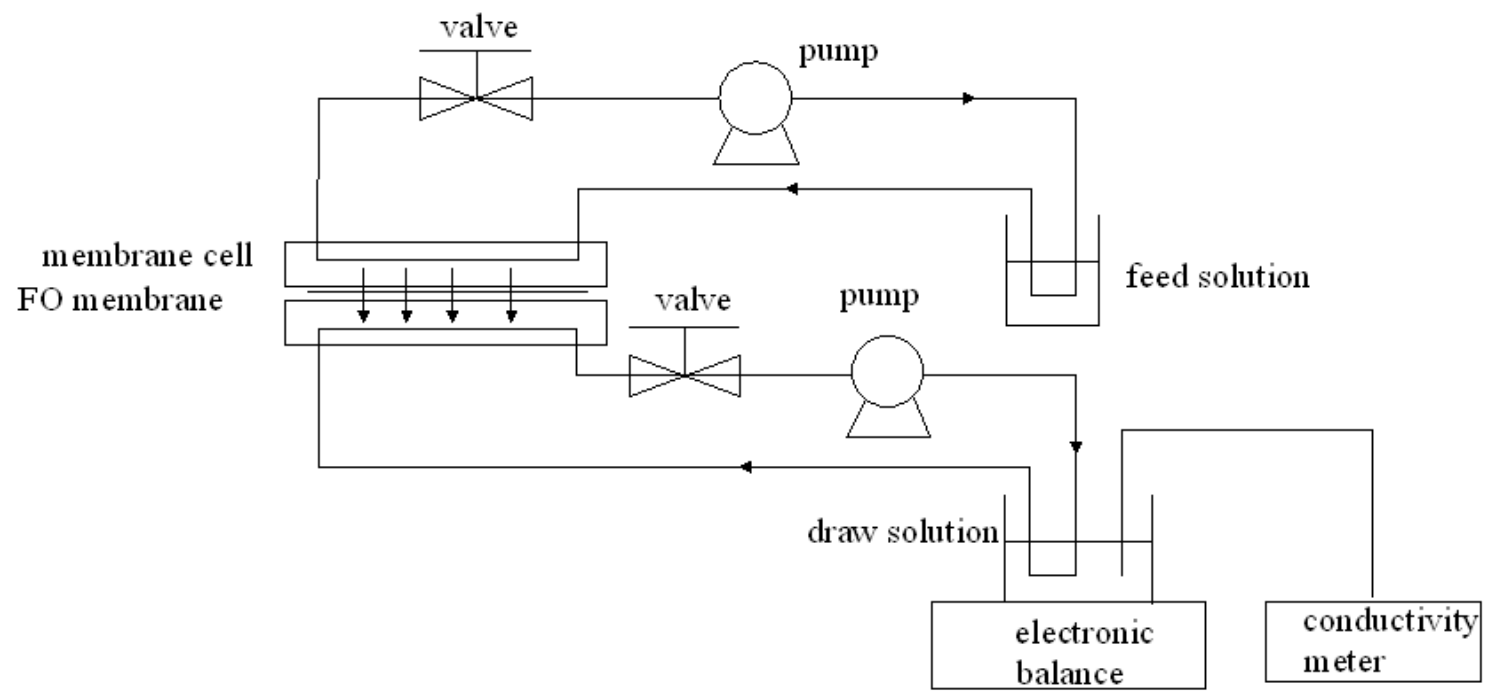

Fig. 1 Laboratory-scale forward osmosis testing device.

The draw solution and feed solution were $4 \mathrm{M}$ glucose and $0.1 \mathrm{M} \mathrm{NaCl}$ solution, respectively. The membrane active layer was contacted with the feed solution (AL-FS). The values of water flux was continuous measured for an hour.

This experiment was repeated three times to yield average data. The water flux $\left(\mathrm{L} / \mathrm{m}^{2} \mathrm{~h}\right)$ is determined from the weight change of the draw solution using a digital mass balance as follows:

$$
J \mathrm{~W}=\Delta m / \rho S \Delta \mathrm{t}
$$

where, $\Delta \mathrm{m}$ is the weight changes of the draw solution in a predetermined time interval $\Delta \mathrm{t}(\mathrm{h}), \rho$ is the water density, $\mathrm{S}$ is the effective membrane area.

The reverse salt flux can determined by measuring the conductivity of the feed using a conductivity meter connected to the computer. The reverse salt flux was determined by the following equation:

$$
\mathrm{Js}=c v / s t
$$

where, Js is the reverse salt flux $\left(\mathrm{g} / \mathrm{m}^{2} \cdot \mathrm{h}\right), \mathrm{c}$ is the $\mathrm{NaCl}$ concentration of the feed solution, $\mathrm{v}$ is the volume of the feed solution, $\mathrm{s}$ is the area of the effective FO membrane, and $\mathrm{t}$ is the running time, respectively.

Characterization of Membranes. Top surface and cross-section images of the membranes were observed using SEM (JEOL Model S4800, Japan). For cross-sectional images, it is necessary that carefully cut the FO membrane using a knife before being mounted on the specimen. The contact angle of FO membrane was measured using a contact angle goniometer (Dataphysics Model OCA40, Germany). 


\section{Results and Discussion}

Effects of GO Content. The effects of GO content on membrane performance were investigated, and Fig. 2 presents water flux and reverse salt flux of $\mathrm{CA} / \mathrm{GO}$ membranes as a function of GO content. We can see that GO content caused positive effect on water flux and negative effect on reverse salt flux at the beginning of the experiment. This could be because of the present of GO conduced the increase of membrane hydrophilcity. The hydrophilicity of the membrane has reverse correlation with surface contact angle. The contact angle of the membranes decreased from $71.6^{\circ}$ to $58.2^{\circ}$ with increasing GO from 0 to $0.15 \mathrm{wt} \%$. However, the repeated addition of GO concentration from $0.1 \mathrm{wt} \%$ to $0.2 \mathrm{wt} \%$ didn't cause an evident effect on the water flux. For reverse salt flux, a strictly negative effect was showed with repeated addition of GO concentration from $0 \%$ to $0.2 \%$. In order to get better membrane performance, the concentration of GO was fixed at $0.15 \mathrm{wt} \%$ in membrane preparation experiments.

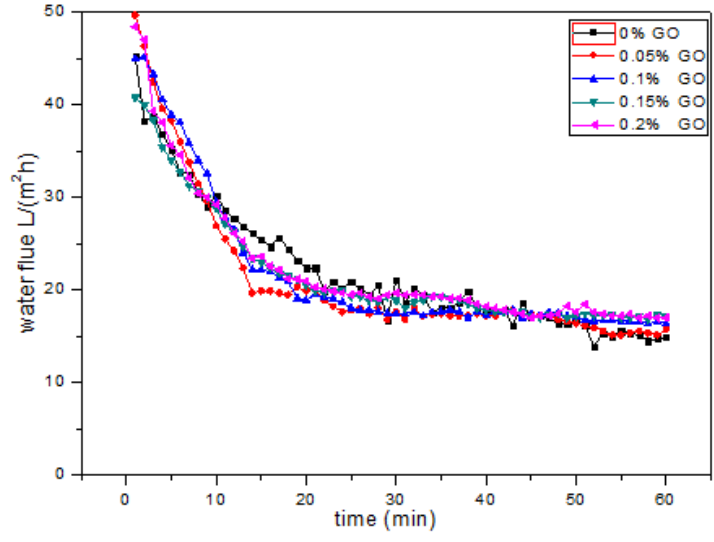

(a)

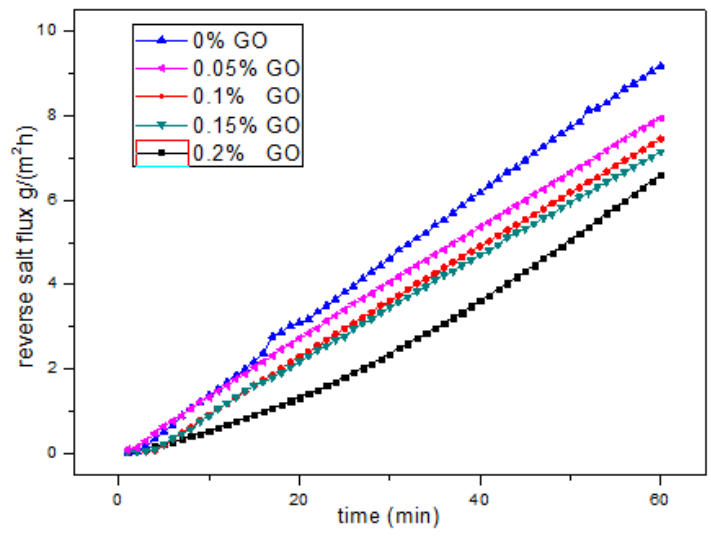

(b)

Fig. 2 The effects of GO content on water flux (a) and reverse salt flux (b).

Effects of Blending Temperature. The effects of blending temperature on FO membrane performance were investigated. From the Fig. 3(a) we can see that the pure water flux of the CA/GO FO membrane increased with the increase of blending temperature, but there is no significant effect on the water flux when the blending temperature increased over $60^{\circ} \mathrm{C}$. Fig. 3(b) showed that the reverse salt flux of the CA/GO FO membrane decreased with the increase of blending temperature at the beginning, and when the temperature increased over $50^{\circ} \mathrm{C}$, the reverse salt flux of the FO membrane increased with the increase of blending temperature. In order to get better $\mathrm{FO}$ membrane performance, the blending temperature was fixed at $50^{\circ} \mathrm{C}$ in membrane preparation experiments.

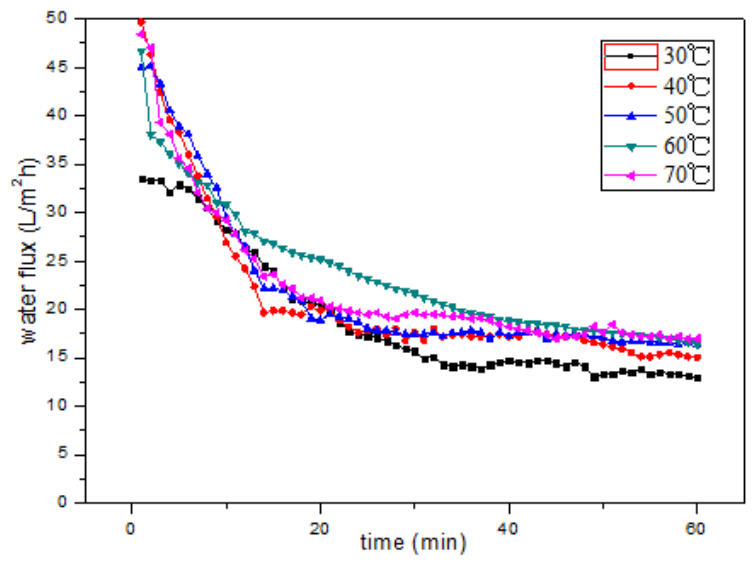

(a)

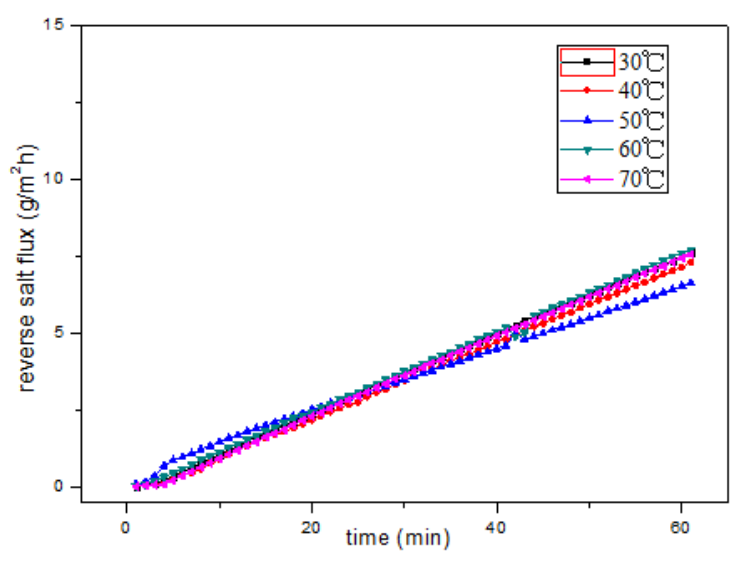

(b)

Fig. 3 The effects of blending temperature on water flux (a) and reverse salt flux (b). 
SEM Image of the Mixed Matrix FO Membrane. The surface and cross section properties of the CA/GO FO membrane were characterized by SEM. As you can see from Fig. 4, the GO was fully dispersed in the CA/GO FO membrane. After blending modification, the FO membrane surface was more smooth, and the polyester mesh screen was clearly visible in the membrane. It can be clearly observed from the cross section of FO membrane that the finger pore structure was more neatly, aperture around 10 to $25 \mu \mathrm{m}$, had obvious big pore structure, and the channel throughout almost the entire section.

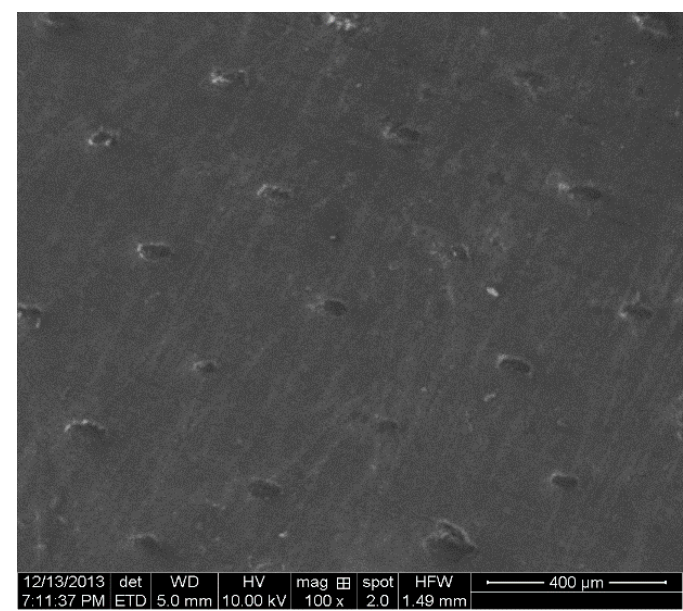

(a) FO membrane surface $(100 \times)$

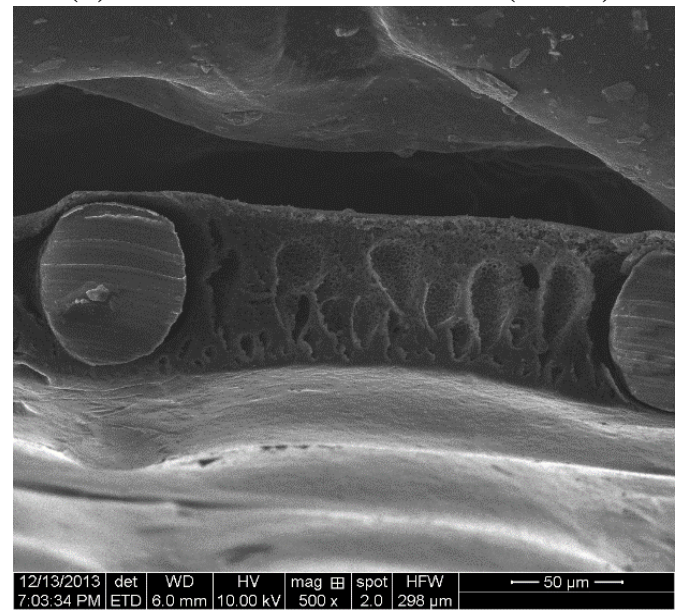

(c) FO membrane cross section (500x)

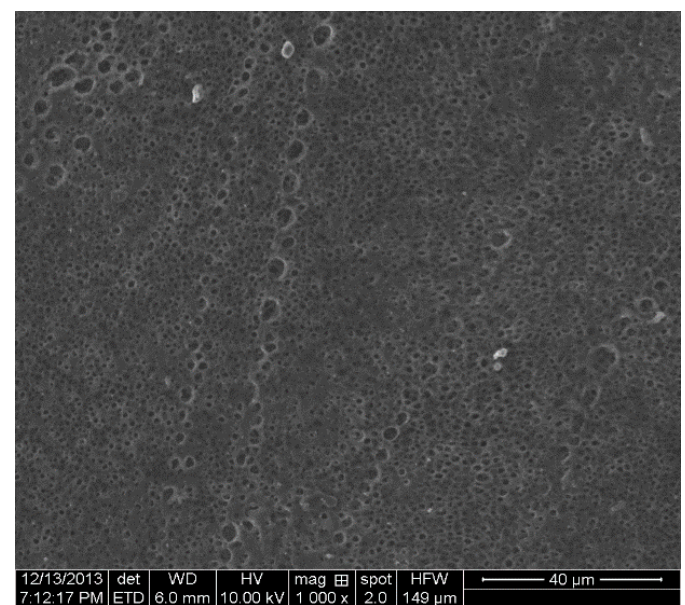

(b) FO membrane surface $(1000 x)$

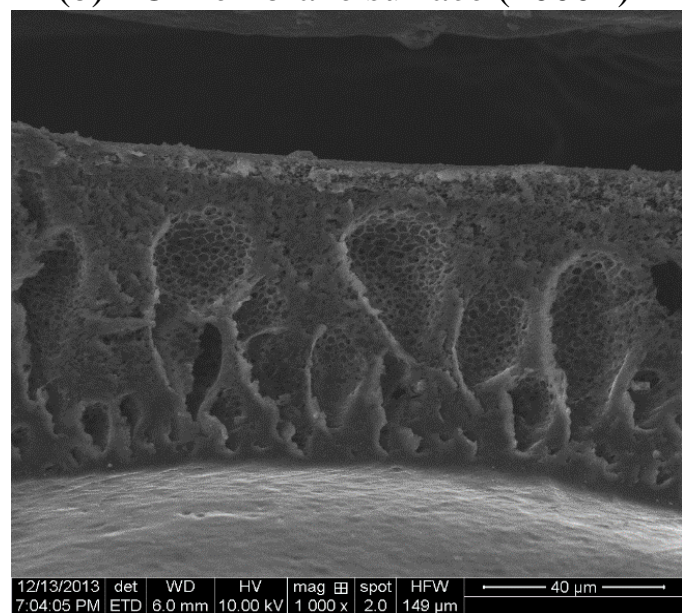

(d) FO membrane cross section (1000x)

Fig. 4 SEM images of surface and cross section of CA/GO FO membrane.

\section{Conclusion}

A novel CA/GO mixed matrix FO membrane was investigated, and the FO membrane blend at $50^{\circ} \mathrm{C}$ with $0.15 \mathrm{wt} \% \mathrm{GO}$ concentration has an optimum performance. The CA/GO mixed matrix FO membrane prepared under optimized composition had excellent desalination performance: high pure water flux $=16.0 \mathrm{~L} / \mathrm{m}^{2} \cdot \mathrm{h}$ and the reverse salt flux was below $7 \mathrm{~g} / \mathrm{m}^{2} \cdot \mathrm{h}$, respectively. SEM images depicted the asymmetric characteristics of membranes with the presence of GO.

\section{Acknowledgements}

This work was financially supported by National Science-Technology Support Plan Projects (2014BAK13B02), Shandong Science and Technology Development Plan (2012SGGX10220), National Natural Science Foundation of Shandong Province (ZR2015BL021), Funds of Key Laboratory of Marine Chemistry Theory and Technology, Ministry of Education. 


\section{References}

[1] Y. Fang, L. Bian, Q. Bi, Evaluation of the pore size distribution of a forward osmosis membrane in three different ways, J. Membr. Sci. 454 (2014) 390-397.

[2] T. P. N. Nguyen, E. T. Yun, I. C. Kim, Preparation of cellulose triacetate/cellulose acetate (CTA/CA)-based membranes for forward osmosis, J. Membr. Sci. 433 (2013) 49-59.

[3] J. R. McCutcheon, M. Elimelech, Influence of membrane support layer hydrophobicity on water flux in osmotically driven membrane processes, J. Membr. Sci. 318 (2008) 458-466.

[4] M. Ghanbari, D. Emadzadeh, W. J. Lau, et al., Super hydrophilic $\mathrm{TiO}_{2} / \mathrm{HNT}$ nanocomposites as a new approach for fabrication of high performance thin film nanocomposite membranes for FO application, Desalination. 371 (2015) 104-114.

[5] M. Amini, M. Jahanshahi, A. Rahimpour, Synthesis of novel thin film nanocomposite (TFN) forward osmosis membranes using functionalized multiwalled carbon nanotubes, J. Membr. Sci. 435 (2013) 233-241.

[6] H. R. Chae, J. Lee, C. H. Lee, et al., Graphene oxide-embedded thin-film composite reverse osmosis membrane with high flux, anti-biofouling, and chlorineresistance, J. Membr. Sci. 483 (2015) 128-135.

[7] Y. Wang, R. Ou, H. Wang, et al., Graphene oxide modified graphitic carbon nitride as amodifier for thin film composite forward osmosis membrane, J. Membr. Sci. 475 (2015) 281-289.

[8] M. Hu, B. Mi, Enabling graphen eoxide nanosheets as water separation membranes, Environ. Sci. Technol. 47 (2013) 3715-3723.

[9] B. Mi, Graphene oxide membranes for ionic and molecular sieving, Sci. 343 (2014) 740-742.

[10] X. Lu, S. Castrillon, D. L. Shaffer, et al., In situ surface chemical modification of thin-film composite forward osmosis membranes for enhanced organic fouling resistance, Environ. Sci. Technol. 47 (2013) 12219-12228.

[11] S. Stankovich, D. A. Dikin, G. H. Dommett, et al., Graphene-based composite materials, Nat. 442 (2006) 282-286.

[12] X. Chang, Z. Wang, S. Quan, et al., Exploring the synergetic effects of graphene oxide (GO) and polyvinylpyrrodione (PVP) on poly (vinylylidenefluoride) (PVDF) ultrafiltration membrane performance, Appl. Surf. Sci. 316 (2014) 537-548.

[13] Y. Wang, T. Xu, A choring hydrophilic polymer in substrate: an easy approach for improving the performance of TFC FO membrane, J. Membr. Sci. 476 (2015) 330-339. 